\title{
A fast bottom-up approach toward three-dimensional human pose estimation using an array of cameras
}

\author{
Maryam Ghaneizad $^{\mathrm{a}}$, Zahra Kavehvash ${ }^{\mathrm{a}, *}$, Khashayar Mehrany ${ }^{\mathrm{a}}$, S.M. Ali Tayaranian Hosseini ${ }^{\mathrm{b}}$ \\ ${ }^{a}$ Department of Electrical Engineering, Sharif University of Technology, Tehran, Iran \\ b Department of Electrical Engineering, Amirkabir University of Technology, Iran
}

\section{A R T I C L E I N F O}

\section{Keywords:}

3D human pose estimation

$3 \mathrm{D}$ reconstruction

Integral imaging

\begin{abstract}
A B S T R A C T
In this paper, employing recorded images of multiple cameras, we propose a novel fast approach for threedimensional (3D) human pose reconstruction. Opening a new framework to the pose estimation application, the proposed method is inspired by optical 3D reconstruction algorithms conventionally used for integral imaging. Thanks to the fact that the pose estimation can be carried out by using only key features of the raw recorded images, the computation time and the complexity of our method are considerably reduced. Furthermore, utilizing the here proposed algorithm, rapid variations in actions can be easily tracked. The validity of the proposed method is demonstrated by several experimental results in different postures.
\end{abstract}

\section{Introduction}

3D human pose estimation is an important problem with many potential applications from gaming and human-computer interaction to smart care centers and surveillance systems [1-3]. The ability to recognize different human postures in real-time can also be very useful for semantic labeling of various environments; that is to perceive the environment in which cameras are built in by extracting the humans' body pose and their activities [4].

Many different methods have been proposed for human pose estimation. Among them, a variety of methods have focused on the $3 \mathrm{D}$ reconstruction of the body pose from monocular image sequences $[5,6]$. Although such methods can successfully recover human pose in different scenarios, there are some situations where the intrinsic inability of monocular cameras to fully explain 3D objects impedes successful reconstruction of human pose. Human body self-occlusion and non-observability of motions along axial direction counts among the most noticeable reasons for the failure of human pose estimation by using monocular image sequences [7]. In recent years, some approaches have been developed based on the depth image analysis. Depth images could provide depth information by encoding an image into different colors [8-13]. For example, in [8-11], depth images provided by Kinect have been used for human detection or pose estimation in indoor environments. Recent approaches on human motion analysis using depth data are reviewed in $[12,13]$. Nevertheless, all methods using depth images suffer from strong noise added to the depth data and also the self-occlusion problem.
There are several other methods [14-16] for human pose estimation, which employ multiple video-streams recorded from different viewing angles and therefore are not burdened with the aforementioned problems. These methods can be generally divided into top-down and bottom-up categories [18].

In top-down methods [14,19-21], a 3D pose model is first assumed, which is usually referred to as the previous time model. A similarity evaluation function is then defined between the 3D model and 2D images. Next, the sought-after 3D model is iteratively obtained as the similarity evaluation function reaches the extremum value [14]. However, searching for the optimal 3D model is a complicated and time-consuming process, which necessitates a nonlinear hierarchical optimization algorithm. Furthermore, the validity of such methods relies heavily upon the validity of time consistency in postures. In other words, in case of relatively rapid body movements, the optimization algorithm will be likely to converge to a local optimum and hence the algorithm fails to lead to the accurate 3D model at the current time [14]. The resultant error can further propagate in future frames' model estimation and end up with a large amount of error.

In bottom-up methods [23-25]; however, features of interest, e.g. silhouettes, edges or body parts, are first detected in 2D images. These features are then assembled into the 3D model. Thus, the accuracy of such methods do not rely on the validity of time consistency in postures. The assembly of the detected features into a certain 3D configuration is a heavy computational task. This computational load may, however, be decreased by using optical imaging principles. To this end, in this paper, for the first time, as far as we know, an optically inspired fast

\footnotetext{
* Corresponding author: Tel.: + 98-9127107687

E-mail address: kavehvash@sharif.edu (Z. Kavehvash).
} 
bottom-up approach for multi-camera image fusion is used in the pose estimation application. Thanks to the fact that the proposed optically inspired 3D image fusion technique doesn't require solving any optimization problem, its time, accuracy and simplicity are much improved compared to conventional top-down frameworks. To the best of our knowledge, the idea of using integral imaging principles has only been utilized in human hand gesture recognition where optical principles are used for the mere purpose of blurring the background [17]. In contrast, the here proposed optically inspired method is employed to provide the 3D human posture which is to be used in whole body gesture analysis.

Given that the proposed approach does not depend on the preceding frames information, any sudden movement of the body can be accurately tracked. Moreover, the speed of pose estimation can be further increased not only because the iterative steps are no longer necessary but also because whole body images conventionally used for pose estimation can be substituted by the image of seed points, i.e. the $3 \mathrm{D}$ location of hands, head and shoulders. The remainder of the paper is structured as follows. Section 2 describes the proposed optical fusion algorithm for human pose estimation. Section 3 discusses the mathematical formulation for our proposed framework. Section 4 confirms the proposed structure through experimental results and finally, Section 5 concludes the paper.

\section{The proposed multi-camera image fusion algorithm}

The proposed framework is based on an intuitive algorithm for fusion of multi-camera images based on closed-form equations used in geometrical optics. The goal is to locate the 3D position of human seed points; hands, head and shoulders, from which the 3D skeleton may be reconstructed. The proposed bottom-up algorithm is very fast and accurate and hence can be a suitable alternative for the complicated vision-based algorithms used for $3 \mathrm{D}$ pose estimation. Having no dependencies on preceding frames information, the model provides two advantages: First, the pose reconstruction is very fast and the algorithm can be implemented in real-time. Second, the algorithm is highly immune to estimation errors and can preclude any probable error from further propagating in successive frames. The proposed algorithm contains the following steps:

(i) Recording multiple 2D images from different viewpoints by ordinary 2D cameras.

(ii) Extracting the seed points on each image based on the image processing algorithm discussed in [18].

(iii) Fusing seed points (images) through optically inspired relations.

(iv) Post-processing the reconstructed $3 \mathrm{D}$ images to derive the $3 \mathrm{D}$ location of seed points and building the final 3D skeleton.

Let us explain each of the aforementioned steps more rigorously.

At the first step of the algorithm, we need to record multiple 2D intensity images from the intended person. To this end, we have proposed a curved arrangement of cameras in addition to the standard linear arrangement. In both arrangements, we are able to use a fairly small number of cameras. Furthermore, both arrangements are onedimensional and no vertical shift is introduced between camera positions. For the curved arrangement, we may further reduce the number of cameras compared to the linear arrangement for a similar performance. This is mainly due to the fact that in the curved arrangement, the diversity between different viewpoint images is increased. Both arrangements will be further discussed in Section 3. The second step is a supplementary step which is included to further improve the efficiency of the proposed algorithm. In our application, i.e. the human pose reconstruction, a lot of information of the human body and the background scene is excessive and needs not to be included in the reconstruction process. Therefore, we have included an image data reduction step in our algorithm to further improve depth resolution, the system speed, the energy consumption and the system available memory. After recording $2 \mathrm{D}$ images by the cameras, each image may be separately processed to yield the only required information for the algorithm which is the positions of so-called seed points. Detection of seed points can be done very easily by the image processing algorithm described in [18]. Doing so enables us to quantize the depth information of the object which lowers down the required depth resolution. This low resolution can be achieved with only a few cameras making the system more cost effective. In other words, using a limited number of cameras, as is the case herein, the depth resolution is very low and without the aforementioned image data reduction, the algorithm will fail to reconstruct depth information. The novelty to remove the excessive information and keep only seed points in $2 \mathrm{D}$ images, helps us to get the most benefit from the available axial resolution. Doing so, we quantize the human body information in 2D images. Since the seed points are apart from each other and the variations in depth are not abrupt, there will remain only a few intensity points with sufficient distances along the axial direction out of a large number of neighboring points with nearly the same depth values. Thus, the available depth resolution, though it is low, will be enough to differentiate these points in the axial direction. Here after, we may call the resultant binary images, containing one at the location of a seed point and zero elsewhere, seed images. Seed images can be represented as the following:

$\widehat{I}_{i}=\sum_{j=1}^{5} \delta\left[x-x_{j}^{i}, y-y_{j}^{i}\right]$

where $\left(x_{j}^{i}, y_{j}^{i}\right)$ are the coordinates (of the image) of $j$ th seed point in the $i$ th seed image, $\delta$ is the 2D Kronecker delta function and five seed points are considered to be the 3D location of head, two shoulders and two hands. At the next step of the algorithm, the proposed optically inspired reconstruction algorithm is used for the fusion of seed images based on the formulation which will be discussed in Section 3. As will be seen there, considering a specific seed point, e.g. head, in all seed images and fusing the images based on optical principles to reconstruct the 3D image at different depth planes, lead to a plain output at depth $z_{0}$ where the head has been originally located and blurred outputs at other depths. The amount of blurring in the reconstructed output at an arbitrary depth plane is proportional to its axial distance from $z_{0}$. Therefore, reconstructing at $z_{0}$, the plain image of the head is accompanied by the adverse blurred images of all other seed points. Although we have drastically reduced this undesirable effect by substituting the whole recorded image at the first step by only a few seed points at the second step, a post-processing is also needed to completely eliminate it.

The final step of the algorithm is the aforementioned post-processing to clear the discussed undesirable effect in reconstructed images and derive a clean 3D image of the 3D locations of seed points, i.e. $\left(x_{j}, y_{j}, z_{j}\right) ; j=1, \ldots, 5$. Joining these virtual seed points together in a volumetric space, we are able to reconstruct the 3D pose of the human.

Thus, the proposed algorithm is a combination of the optically inspired relations and the image processing algorithms to further improve the efficiency and the performance of the pose estimation algorithm. The detailed formulation of the proposed algorithm for both mentioned camera configurations will be discussed in the next section.

\section{Mathematical formulation}

In this section, the proposed optically inspired multi-camera image fusion technique is mathematically formulated. Let us consider the viewpoint images recorded by each camera as $I_{i}\left(x^{\prime}, y^{\prime}\right) ; i=1, \ldots, N$, with $x^{\prime}$ and $y^{\prime}$ in the range of the image size and $\mathrm{N}$ the total number of cameras and let us represent the seed images as $\hat{I}_{i}\left(x^{\prime}, y^{\prime}\right)$ each being a sparse binary matrix. The seed images are the required input for the reconstruction process. In this process, $2 \mathrm{D}$ positions of each seed point 
in different viewpoint images contribute together to reconstruct the 3D position of that body part in a virtual space. In this section, we will follow the procedure in which volumetric reconstruction is done mathematically. First, consider the recording process shown for different camera arrangements in Fig. 1, in which a number of 2D images are recorded from the standing human. In this process, each voxel of the human body at position $(x, y, z)$ is mapped to the image plane of each camera. In other words, each voxel $(x, y, z)$ contributes to form a number of pixels each on a different image plane. Due to the light propagation, the optical intensity falls down relative to the squared value of traveling distance, $d_{i}$, thus [33]:

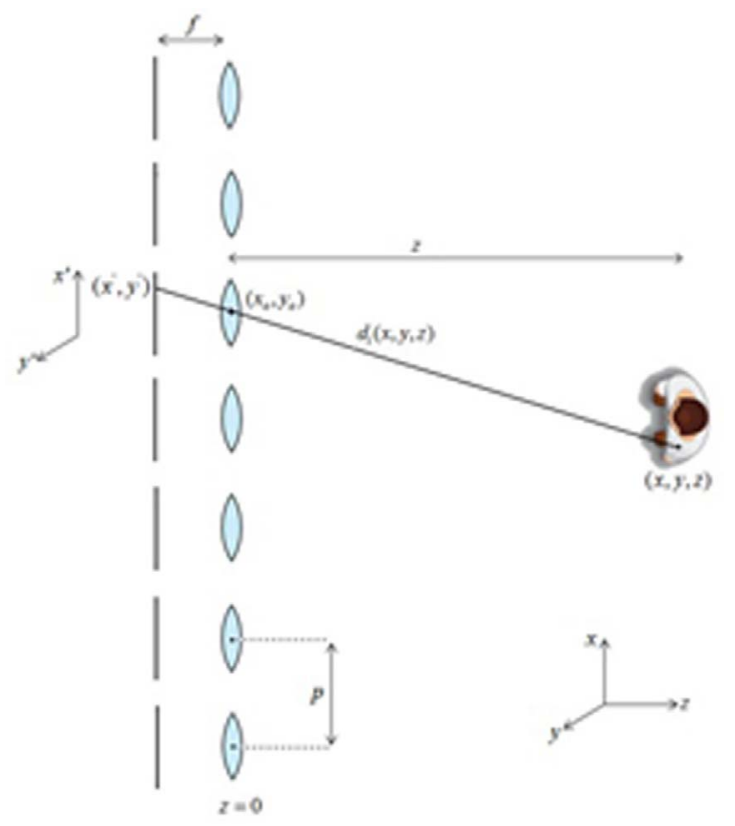

(a) Linear arrangement

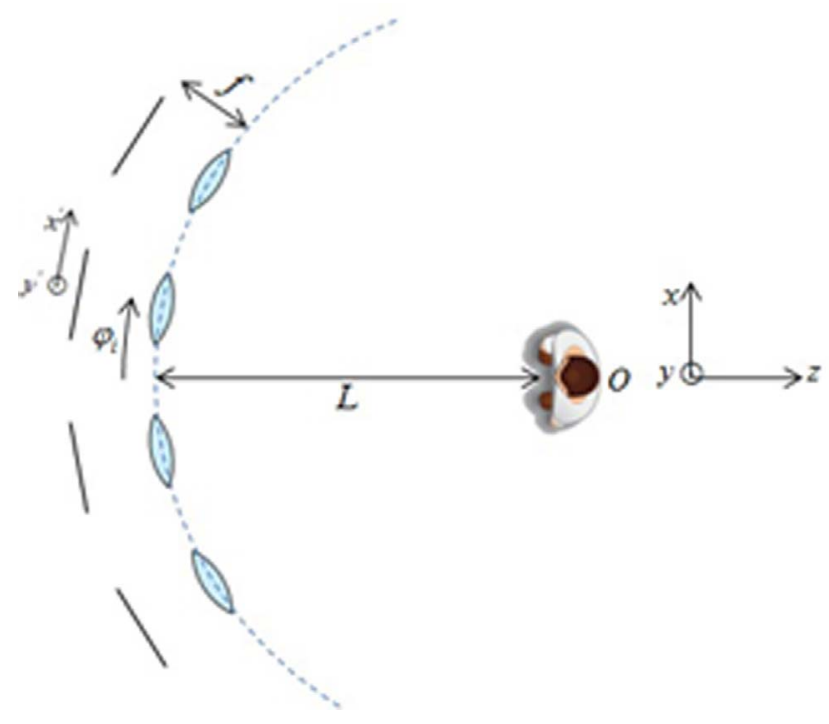

(b) Curved arrangement

Fig. 1. The schematic depiction of recording step in the optical fusion algorithm for different camera configurations.
$\widehat{I}_{i}\left(x^{\prime}, y^{\prime}\right)=\frac{O(x, y, z)}{d_{i}^{2}(x, y, z)} \quad \forall i=1, \ldots, N$

where $(x, y, z)$ is assumed to be a 3D point on the human body and $\left(x^{\prime}, y^{\prime}\right)$ is the corresponding 2D point on the image plane of the $i$ th camera. Based on a well-known principle in optics called light reversibility principle, the computational reconstruction can be considered as the inverse process of the recording process. Using this fact, in the following, we derive the mathematical expressions for the computational reconstruction process separately for each of the linear and curved camera arrangements.

\subsection{Linear arrangement}

For the linear arrangement of the cameras, the transformation from the 3D space to 2D image planes can be defined as:

$\left\{x^{\prime}=x_{o}-\left(x-x_{o}\right) \frac{f}{z}\right.$

$y^{\prime}=y_{o}-\left(y-y_{o}\right) \frac{f}{z}$

where $\left(x_{o}, y_{o}\right)$ are the camera lens centroid coordinates in the transverse plane, $f$ is the lens gap set equal to the focal length of the lenses as is a common setting in the focal mode imaging. In the horizontal linear camera arrangement, the lens centroid coordinates for the ith camera are easily found to be:

$\left\{x_{o}=\left(i-\frac{N+1}{2}\right) p \quad \forall i=1, \ldots, N\right.$

$y_{o}=0$

where $N$ and $p$ are the number of cameras and lens pitch, respectively. Using Eqs. (3) and (4), the light traveling distance from the virtual 3D point $(x, y, z)$ on the human body to the corresponding point on the $i$ th 2D image $\left(x^{\prime}, y^{\prime}\right)$ can be written as:

$d_{i}^{2}(x, y, z)=\left(x-x^{\prime}\right)^{2}+\left(y-y^{\prime}\right)^{2}+(z+f)^{2}=$

$\left\{\left[x-\left(i-\frac{N+1}{2}\right) p\right]^{2}+y^{2}+z^{2}\right\}\left(1+\frac{f}{z}\right)^{2}$

Now the value of the voxel $(x, y, z)$ can be computed based on the value of the corresponding pixels on different image planes. A portion of this value can be obtained by back-projection of the pixel $\left(x^{\prime}, y^{\prime}\right)$ located on the $i$ th $2 \mathrm{D}$ image:

$O_{i}(x, y, z)=\widehat{I}_{i}\left(x^{\prime}, y^{\prime}\right) \cdot d_{i}^{2}(x, y, z)$

Eq. (6) can be rewritten using Eqs. (3)-(5) as:

$O_{i}(x, y, z)=\left\{\left[x-\left(i-\frac{N+1}{2}\right) p\right]^{2}+y^{2}+z^{2}\right\}\left(1+\frac{f}{z}\right)^{2}$.

$\widehat{I}_{i}\left(\left(i-\frac{N+1}{2}\right) p-\left[x-\left(i-\frac{N+1}{2}\right) p\right] \frac{f}{z},-y \frac{f}{z}\right)$

Summing the effect of all back-projected 2D images in the 3D space, we obtain:

$O(x, y, z)=\sum_{i} O_{i}(x, y, z)$

which yields the 3D image. According to the light reversibility principle, we claim that every seed point on a seed image introduces a set of virtual seed points in the 3D space all located on a line emanating from the 2D seed point location through the corresponding lens centroid, as shown in Fig. 2. This assertion can be easily proved using the formulation provided in this section. To be more specific, 


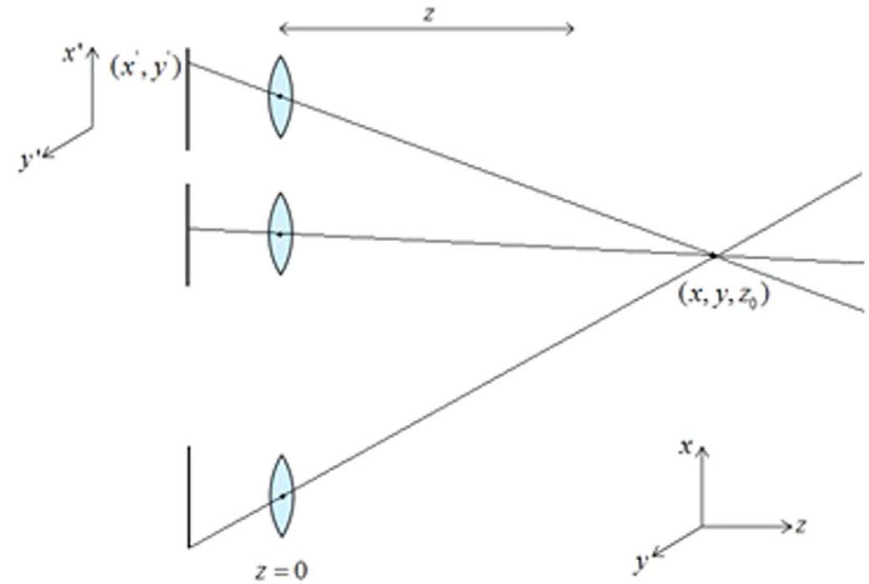

Fig. 2. 3D reconstruction of an infinitesimal point source on the human body in the linear camera arrangement.

Table 1

Parameters for three different postures of the manikin in the linear arrangement scenario.

\begin{tabular}{llll}
\hline & $z_{H S}(\mathrm{~cm})$ & $z_{R}(\mathrm{~cm})$ & $z_{L}(\mathrm{~cm})$ \\
\hline Posture \#1 & 43 & 48 & 37.5 \\
Posture \#2 & 42 & 36 & 42 \\
Posture \#3 & 35 & 29 & 29 \\
\hline
\end{tabular}

rearranging Eq. (3), we obtain:

$\left\{x=x_{o}+\frac{z}{f}\left(x_{o}-x^{\prime}\right)\right.$

$y=-y^{\prime}$

which introduces the locus of a line corresponding to the seed point $\left(x^{\prime}, y^{\prime}\right)$ for a specific lens coordinate $\left(x_{o}, 0\right)$ where the position of the virtual 3D seed point on the line depends on the reconstruction depth $z$. Considering all lines corresponding to a specific seed point, they continue their path to converge at the point where the 3D seed point has been originally located and then start diverging. Therefore, the plain image of the seed point can only be observed at the depth plane of

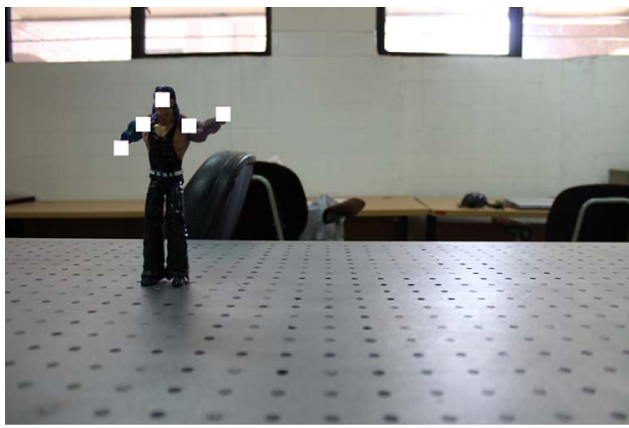

Fig. 4. Seed points marked on the first camera image.

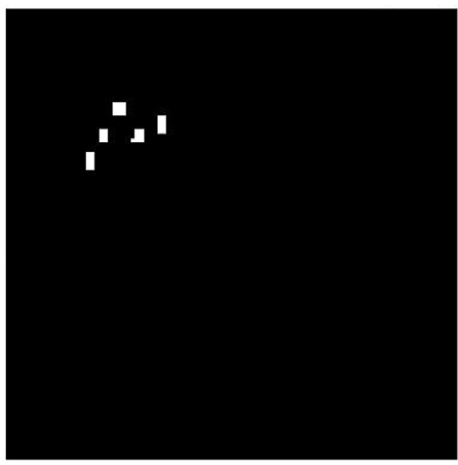

Fig. 5. The first camera seed image.

its original location while a blurred image of it can be seen elsewhere. This differentiation can help in finding the depth location of the 3D seed point.

\subsection{Curved arrangement}

For the curved arrangement of the cameras, it can be shown through geometrical relations that the relationship between the 3D point $(x, y, z)$ and 2D image point $\left(x^{\prime}, y^{\prime}\right)$ on the ith $2 \mathrm{D}$ image can be written as:
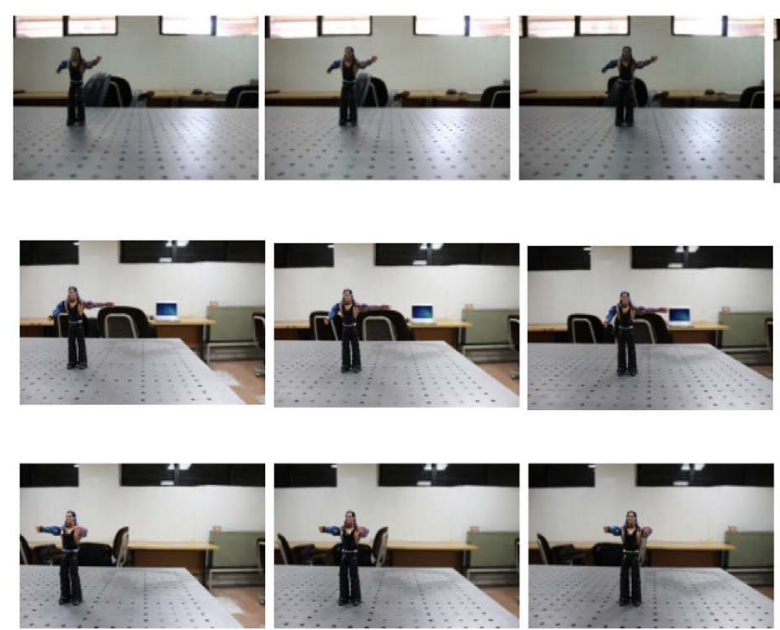
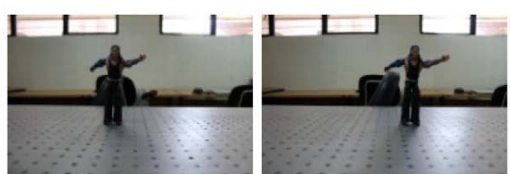

Posture \#1
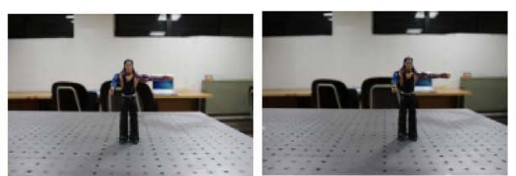

\section{Posture \#2}
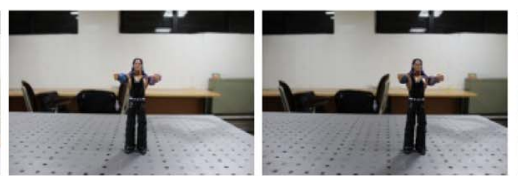

\section{Posture \#3}
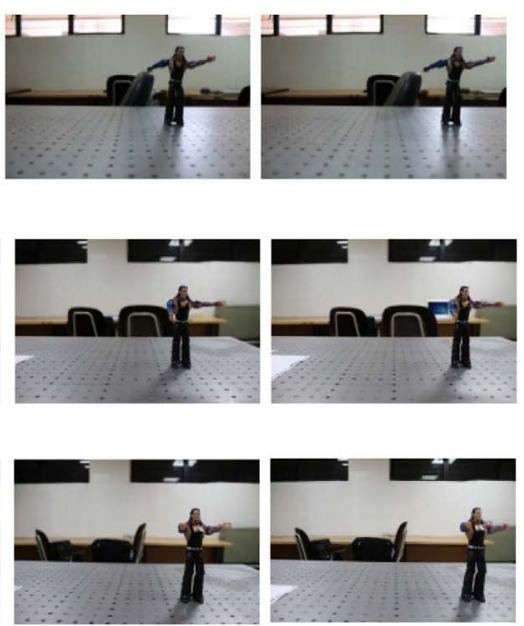

Fig. 3. Different viewpoint images for three postures in the linear arrangement scenario. 


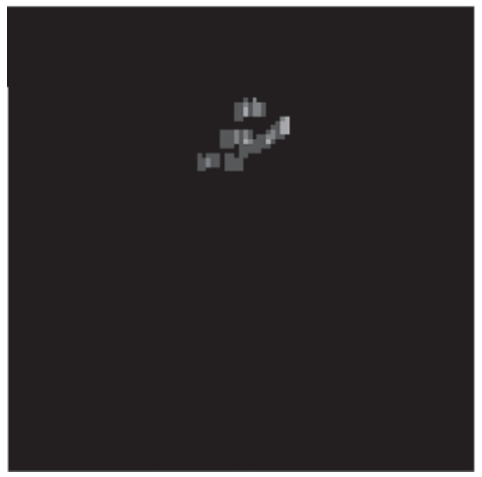

(a) $z=z_{L}$

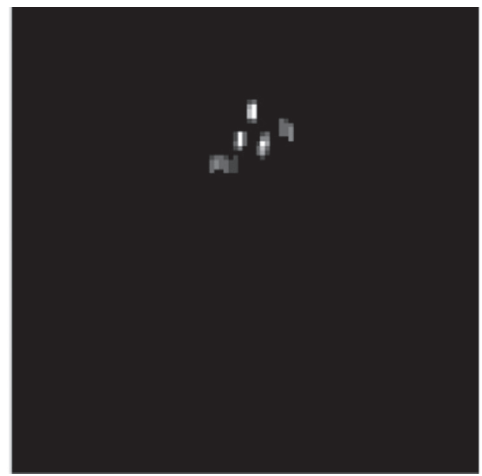

(b) $z=z_{H S}$

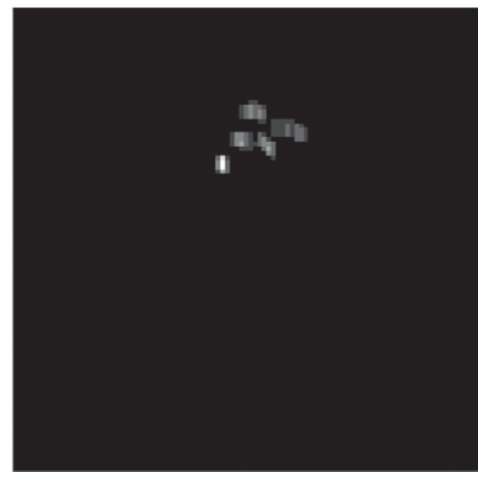

(c) $z=z_{R}$

Fig. 6. 3D computationally reconstructed images at depth planes $z=z_{L}, z_{H S}, z_{R}$ for the first posture in the linear arrangement scenario.

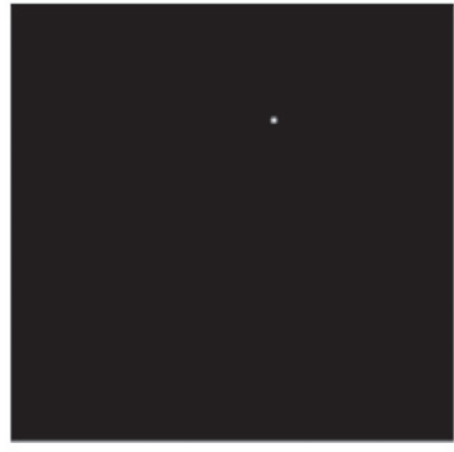

(a) $z=z_{L}$

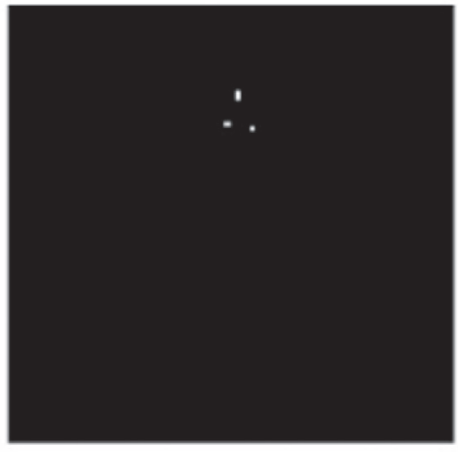

(b) $z=z_{H S}$

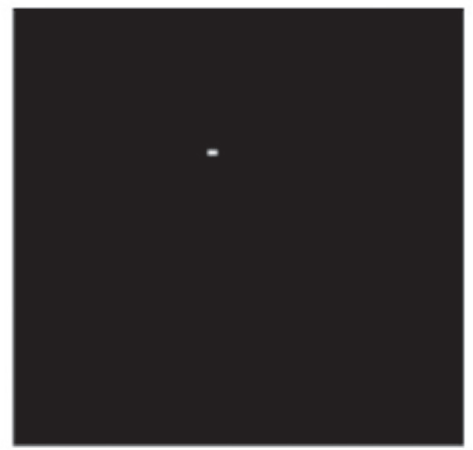

(c) $z=z_{R}$

Fig. 7. Clean images derived by post-processing at depth planes $z=z_{L}, z_{H S}, z_{R}$ for the first posture in the linear arrangement scenario

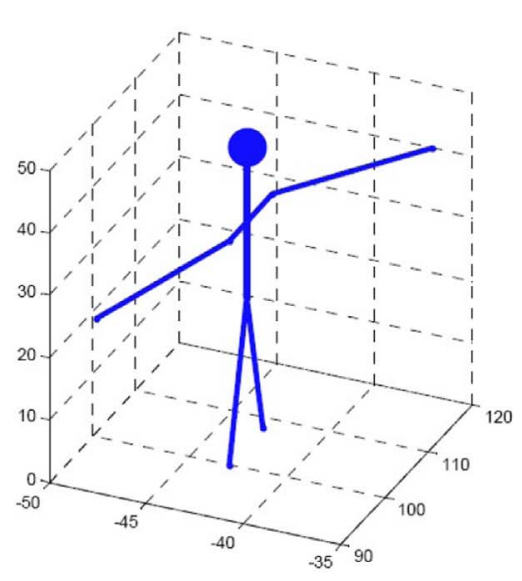

(a) Posture \#1

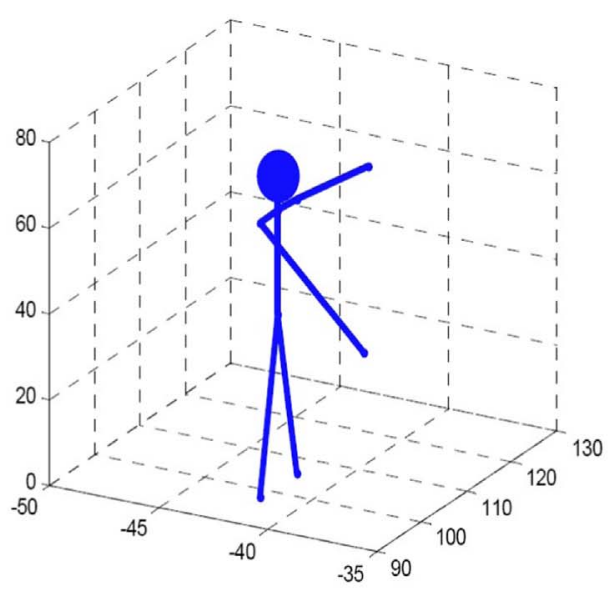

(b) Posture \#2

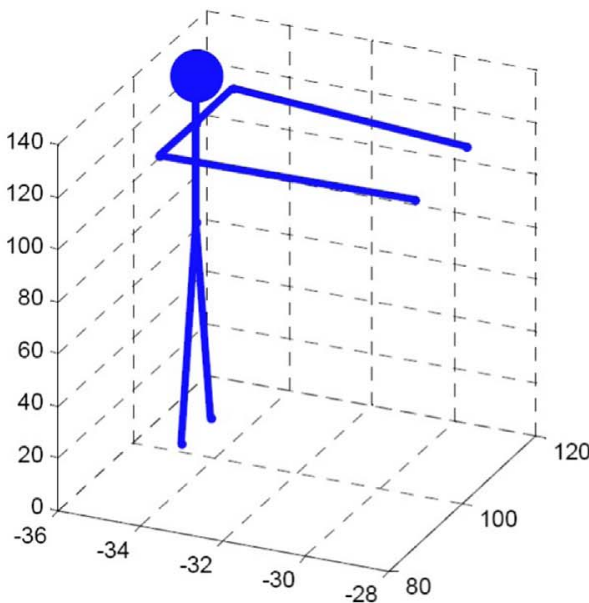

(c) Posture \#3

Fig. 8. The reconstructed 3D skeletons for the postures considered in the linear arrangement scenario. 
Table 2

Parameters for three different postures of the manikin in the curved arrangement scenario.

\begin{tabular}{llll}
\hline & $z_{H S}(\mathrm{~cm})$ & $z_{R}(\mathrm{~cm})$ & $z_{L}(\mathrm{~cm})$ \\
\hline Posture \#1 & -0.5 & -4.9 & -8 \\
Posture \#2 & -0.9 & -4.1 & -7.5 \\
Posture \#3 & 0 & -2.4 & -6.5 \\
\hline
\end{tabular}

$$
\begin{aligned}
& \left\{x=|z| \tan \phi_{i}-\frac{x^{\prime} \cos \phi_{i}\left(L-\frac{|z|}{\cos \phi_{i}}\right)}{f}-\frac{x^{\prime} \sin \phi_{i}\left(L-\frac{|z|}{\cos \phi_{i}}\right)}{f \cdot \tan \left(\tan ^{-1}\left(\frac{f}{x^{\prime}}\right)-\phi_{i}\right)}\right. \\
& y=-\frac{y^{\prime}}{f}\left[L+\sin \phi_{i}\left(\frac{x^{\prime} \cos \phi_{i}\left(L-\frac{|z|}{\cos \phi_{i}}\right)}{f}+\frac{x^{\prime} \sin \phi_{i}\left(L-\frac{|z|}{\cos \phi_{i}}\right)}{f \cdot \tan \left(\tan ^{-1}\left(\frac{f}{x^{\prime}}\right)-\phi_{i}\right)}\right)\right. \\
& \left.-\frac{|z|}{\cos \phi_{i}}\right]
\end{aligned}
$$

where $L$ is the radius of the circle on the periphery of which camera lenses are located, $f$ is again the lens gap and $\phi_{i}$ is the viewing angle of the ith camera shown in Fig. 2. The light traveling distance $d_{i}(x, y, z)$ from the virtual 3D point $(x, y, z)$ to the corresponding 2D point $\left(x^{\prime}, y^{\prime}\right)$ can again be calculated following simple trigonometric relations. Thus, the intensity of the voxel $(x, y, z)$ can be found as:

$O(x, y, z)=\sum_{i} O_{i}(x, y, z)=\sum_{i} \hat{I}_{i}\left(x^{\prime}, y^{\prime}\right) \cdot d_{i}^{2}(x, y, z)$

As explained before, at the depth plane of existence of each seed point,
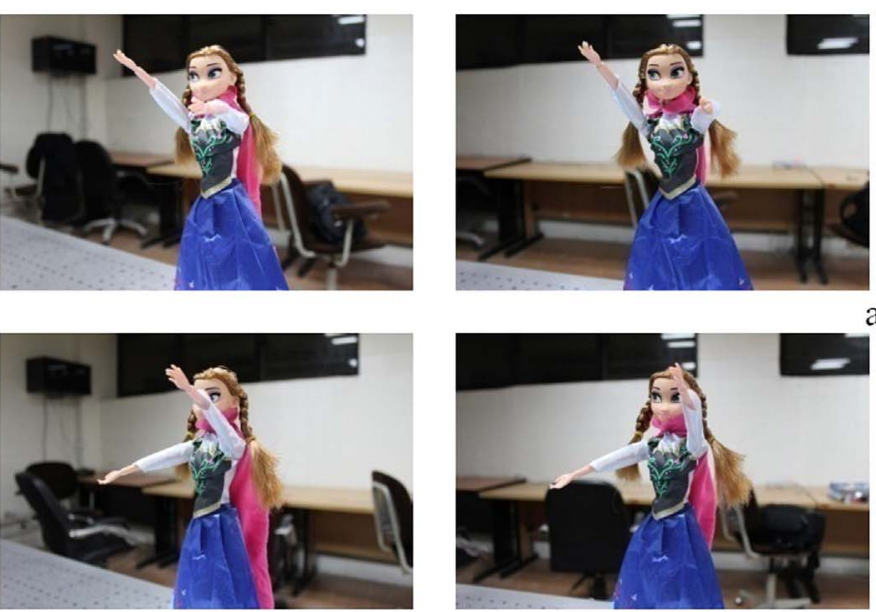

a)

a) Posture \#1
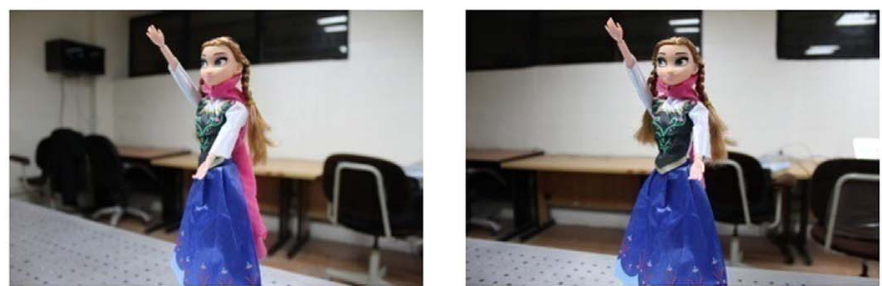

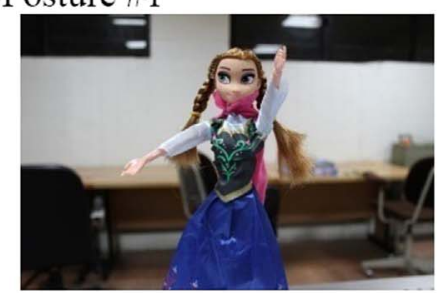

\section{b) Posture \#2}

that specific seed point can be clearly observed with a high intensity value while the other seed points, not located in the same range, may appear as low-intensity speckles. Consequently, a post-processing step is required to eliminate the speckles and result in clean images specifying the exact 3D location of seed points. The extracted seed points can be placed in the 3D space to make the final 3D skeleton.

The proposed algorithm has been experimentally validated in the following section. In the experiments, it is assumed, without loss of the generality, that body motion is intended to be tracked in the upper part of the body and legs are in a fixed position which is a logical assumption for the common application of (upper body) gesture recognition. The legs positions can also be included in the algorithm for other possible applications, such as fall detection or the environment discovery based on human activities.

\section{Experimental results}

Our experimental setup consists of a manikin placed on the optical table and a camera recording the scene from a number of different viewpoints. The camera position has been shifted on the optical table correspondingly to realize both a linear arrangement of cameras (as the first experiment) and a curved one (as the second experiment). The manikin has been positioned in three different poses for each experiment and the computational 3D image reconstruction has been performed based on the mathematical formulation discussed in Section 3. The recorded viewpoint images have been utilized to reconstruct the 3D skeleton of the manikin and illustrate its body pose at any specific posture. In our first experiment, as will be discussed in Section 4.1, a linear array of cameras (camera positions) including seven cameras has been used. In the second experiment, a curved array of cameras including four cameras has been utilized while its corresponding results will be given in Section 4.2. This minimum number of cameras in both arrangements has been chosen through performing several experiments on different human postures and extracting the 3D skeleton in each case. The lowest number of cameras yielding accurate results in all experiments, has been chosen as the minimum number of cameras for each camera arrangement.
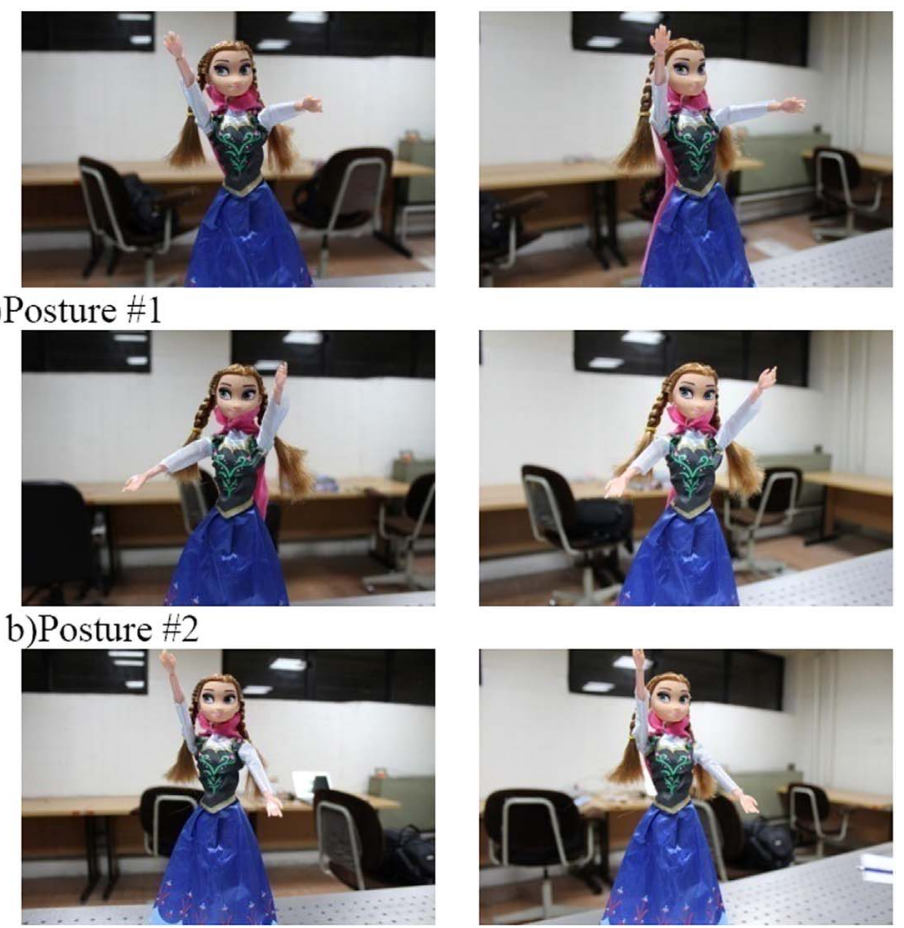

c)Posture \#3

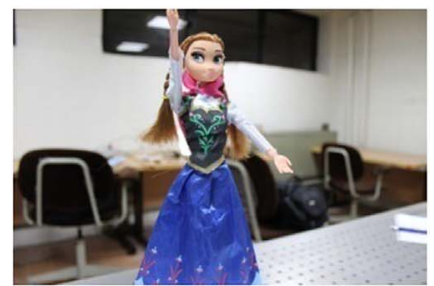

Fig. 9. Different viewpoint images for three postures in the curved arrangement scenario. 


\subsection{First experiment: linear arrangement}

For the linear arrangement of the cameras, as depicted in Fig. 1(a), seven camera positions with a uniform shifting step of $\Delta x=5 \mathrm{~cm}$ have been selected such that the fourth camera is oriented at line-of-sight to the manikin with three camera positions at its right side and three at its left side. As depicted in Fig. 1(a), the $x$ axis is chosen to be the camera shifting line, $z$ axis to be the optical axis of the camera and $y$ axis to be perpendicular to the optical table's surface. The origin coincides with the fourth camera position. The manikin has been put at three different postures with the head and shoulders lying in the same depth plane. However, hands have been arbitrarily moved to lie in different depth planes. Therefore, in general, three different depth planes can be considered corresponding to head and shoulders, right hand and left hand. We refer to these depth planes as $z_{H S}, z_{R}, z_{L}$, respectively. These parameters, set differently for each posture, are mentioned in Table 1. The camera position has been shifted by a step of $\Delta x=5 \mathrm{~cm}$ and seven different viewpoint images have been recorded at each pose of the manikin. These images are shown in Fig. 3 for all postures. At the next step, the coordinates of seed points, head, shoulders and hands, should be determined on each camera image. The seed points, marked as small white rectangles, are shown in Fig. 4 for the first camera image. The seed images, specifying the location of seed points, contain the minimal

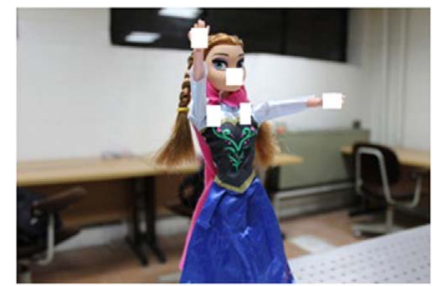

Fig. 10. Seed points marked on the fourth camera image.

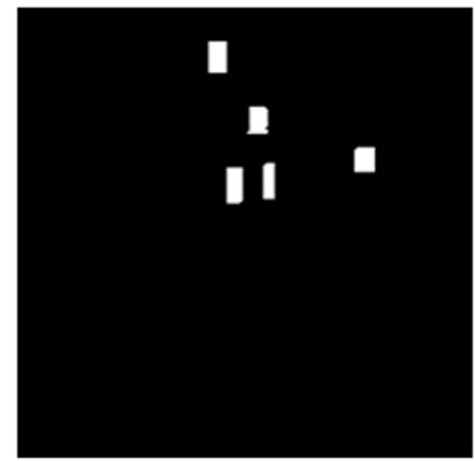

Fig. 11. The fourth camera seed image.

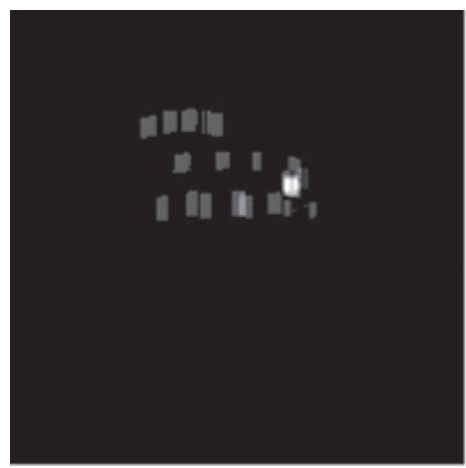

(a) $z=z_{L}$ information needed for the reconstruction of the manikin's pose. The first camera seed image is shown in Fig. 5.

At the reconstruction step, the seed images are fused together to form the 3D image at any arbitrary depth plane based on Eqs. (7) and (8). The results of the 3D computational reconstruction at depth planes $z=z_{L}, z_{H S}, z_{R}$ are shown in Fig. 6 for the first posture.

As apparently seen in Fig. 6, the left hand marker is observed in focus in the left image, while the other seed points are out of focus. In the middle image, the head and shoulders are clearly seen and the speckles are related to the hands. In the right image, however, the right hand can be easily distinguished from the other blurred parts.

The results in Fig. 6 are then post-processed to lead to clean images containing only the fully-overlapped seed points. The required postprocessing has been performed through using open and close algorithms (dilation and erosion) which are well-known image processing algorithms for image denoising. The results of post-processing on the reconstructed images are shown in Fig. 7.

Based on these clean images, each seed point's 3D position is determined. Hence, by displaying the three depth planes in a volumetric space and linking 3D seed points, the 3D skeleton of the human is plotted. The reconstructed 3D skeletons for three different postures of the manikin, listed in Table 1, are shown in Fig. 8.

\subsection{Second experiment: curved arrangement}

For the improved curved camera arrangement, as depicted in Fig. 1(b), four camera positions have been considered uniformly on the periphery of a circle of radius $L=16 \mathrm{~cm}$ and at angles $\phi=-30^{\circ},-10^{\circ}, 10^{\circ}, 30^{\circ}$ with respect to the center of the circle. The manikin has been placed at the center of the circle and fronted to the observer located at $\phi=0$. The object 3D coordinate system and each image plane 2D coordinates are selected as shown in Fig. 1(b).

Following the same steps as the previous experiment, the manikin has been put at three different postures. For the upright postures considered here, the aforementioned assumption for head and shoulders to be coplanar in the depth direction is still valid. For each posture of the manikin, $z_{H S}, z_{R}$ and $z_{L}$ are shown in Table 2. Four different viewpoint images recorded at each posture of the manikin are shown in Fig. 9. Seed points then have to be detected on each camera image. The typical result for the fourth camera image is shown in Fig. 10. The corresponding seed image is also shown in Fig. 11. Seed images have been obtained for all camera images in a similar manner and have been used as the input to the 3D reconstruction step.

At the reconstruction step, seed images may be fused together to form the 3D image at any arbitrary depth plane based on Eqs. (10) and (11). The results of 3D computational reconstruction at depth planes $z=z_{L}, z_{H S}, z_{R}$ are shown in Fig. 12 for the first posture.

The aforementioned post-processing is done using the same technique as the linear arrangement to extract in-focus seed points at each

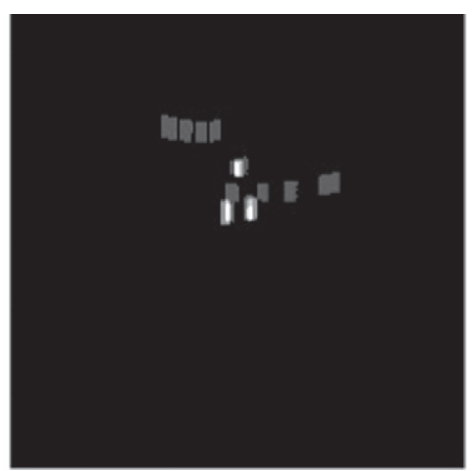

(b) $z=z_{H S}$

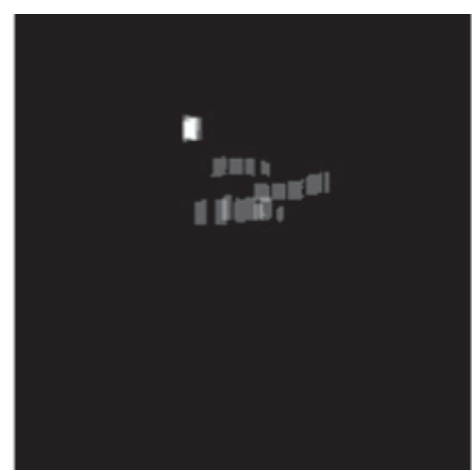

(c) $z=z_{R}$

Fig. 12. 3D computationally reconstructed images at depth planes $z=z_{L}, z_{H S}, z_{R}$ for the first posture in the curved arrangement scenario. 


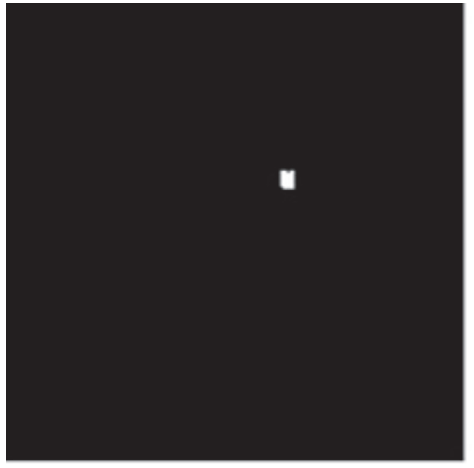

(a) $z=z_{L}$

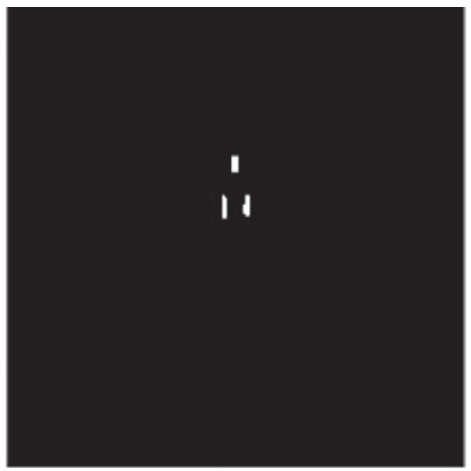

(b) $z=z_{H S}$

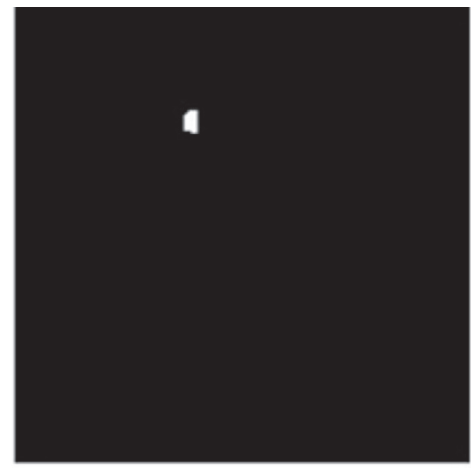

(c) $z=z_{R}$

Fig. 13. Clean images derived by post-processing at depth planes $z=z_{L}, z_{H S}, z_{R}$ for the first posture in the curved arrangement scenario.

depth plane. The results obtained for the first posture are shown in Fig. 13.

Finally, illustrating these clean images in a volumetric space, the 3D skeleton of the manikin has been reconstructed. The reconstructed 3D skeletons for three different postures of the manikin, listed in Table 2, are shown in Fig. 14.

In the proposed 3D skeleton reconstruction method, independent of the camera arrangement, there is no operation with high complexity. Besides, given that the required computations for each pixel are independent of the other pixels, they can be calculated simultaneously using digital parallel processing. Therefore, the reconstruction process, when implemented on a digital hardware, can be performed in a few tens of milliseconds verifying the ability of our algorithm to be utilized in real-time applications. As verified by the experiments, human postures can be accurately estimated by fusing a set of recorded viewpoint images through the proposed optical fusion algorithm. Using the closed-form relations presented in this paper, rather than conventional iterative optimizations, makes our approach considerably fast and accurate. Furthermore, the novelty to use both seed point images and an improved curved camera arrangement has decreased the number of required cameras to be as low as four cameras for a typical scene.

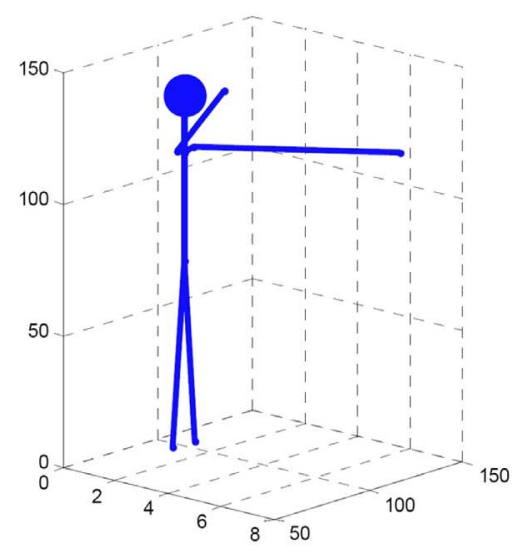

(a) Posture \#1

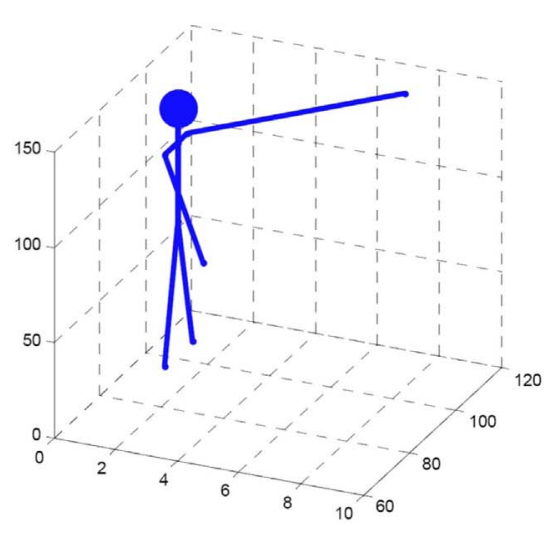

(b) Posture \#2

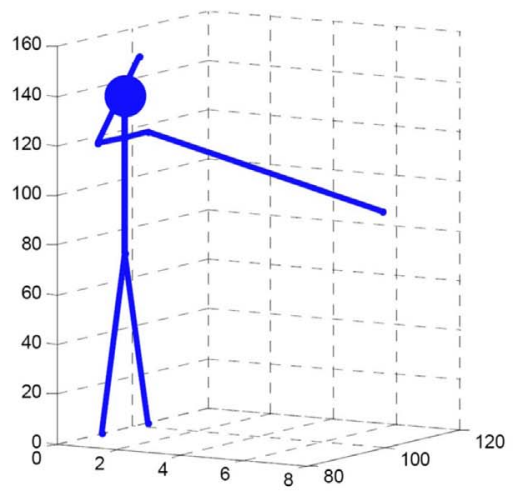

(c) Posture \#3

Fig. 14. The reconstructed 3D skeletons for the postures considered in the curved arrangement scenario. 


\section{Conclusion}

In this paper, we have presented an accurate and fast method for multi-camera 3D pose estimation. In our method, multiple viewpoint images are recorded by an ordinary camera and then processed by an efficient optical method to reconstruct the 3D human posture. An improved curved arrangement of the cameras has been proposed and experimentally verified, as well as the conventional linear camera arrangement. The efficiency of our human pose reconstruction algorithm stems from two main improvements made to the previous methods: First, we have reduced the data recorded by the camera to the minimal essential information through using seed points playing a significant role in human pose estimation instead of raw images. This data reduction not only has considerably increased the algorithm speed, but also has lowered down the demanded depth resolution and hence the required number of cameras. Second, utilizing closed-form optical relations, the proposed algorithm is independent of preceding frames' information which further improves the speed and accuracy. The experimental results, obtained on two manikins, demonstrate the effectiveness of our method to accurately reconstruct the 3D human pose for both linear and curved arrangements. For the curved arrangement, however, we have been able to reduce the number of cameras by two times for a similar performance as the linear setup. Three postures of the manikin have been successfully reconstructed in each experiment. The proposed algorithm has a high potential to be used in several real-time applications, including assisted living and environment discovery based on human activities.

\section{Acknowledgments}

Zahra Kavehvash would like to thanks Iran National Science Foundation for their supports under the contract number of 93031713.

\section{References}

[1] Hilton Adrian, et al. Whole-body modelling of people from multiview images to populate virtual worlds. Vis Comput 2000;16(7):411-36.

[2] Tabar Ali Maleki, Keshavarz Arezou, Aghajan Hamid. Smart home care network using sensor fusion and distributed vision-based reasoning. Proceedings of the 4th ACM international workshop on Video surveillance and sensor networks. ACM 2006

[3] Aghajan Hamid, et al. Distributed vision-based accident management for assisted living. International conference on Smart homes and health telematics. Springer Berlin Heidelberg; 2007.

[4] Wu Chen, Aghajan Hamid. User-centric environment discovery with camera networks in smart homes. IEEE Trans Syst Man Cybern-Part A: Syst Hum
2011:41(2):375-83.

[5] Sidenbladh Hedvig, Michael J Black, David J Fleet. Stochastic tracking of 3D human figures using 2D image motion. Computer vision-ECCV 2000. Berlin Heidelberg: Springer; 2000. p. 702-18.

[6] Deutscher Jonathan, Blake Andrew, Reid Ian. Articulated body motion capture by annealed particle filtering. Computer vision and pattern recognition, 2000. Proceedings. IEEE conference on, vol. 2. IEEE; 2000.

[7] Sminchisescu Cristian, Triggs Bill. Kinematic jump processes for monocular 3D human tracking. Computer vision and pattern recognition, 2003. Proceedings. 2003 IEEE computer society conference on, vol. 1. IEEE; 2003.

[8] Plagemann Christian, et al. Real-time identification and localization of body parts from depth images. Robotics and automation (ICRA), 2010 IEEE international conference on. IEEE; 2010.

[9] Xia Lu, Chia-Chih Chen, Aggarwal Jake K. Human detection using depth information by Kinect. Computer vision and pattern recognition workshops (CVPRW), 2011 IEEE computer society conference on. IEEE; 2011.

[10] Wei Xiaolin, Zhang Peizhao, Chai Jinxiang. Accurate realtime full-body motion capture using a single depth camera. ACM Trans Graph (TOG) 2012;31(6):188.

[11] Shotton Jamie, et al. Real-time human pose recognition in parts from single depth images. Commun ACM 2013;56(1):116-24.

[12] Ye Mao, et al. A survey on human motion analysis from depth data. In: Time-offlight and depth imaging. Sensors, algorithms, and applications. Berlin, Heidelberg: Springer; 2013. p. 149-87.

[13] Chen Lulu, Wei Hong, Ferryman James. A survey of human motion analysis using depth imagery. Pattern Recognit Lett 2013;34(15):1995-2006.

[14] Wu Chen, Aghajan Hamid. Model-based human posture estimation for gesture analysis in an opportunistic fusion smart camera network. Advanced video and signal based surveillance, 2007. AVSS 2007. IEEE conference on. IEEE; 2007.

[15] Blan Alexandru O, et al. Detailed human shape and pose from images. Compute vision and pattern recognition, 2007. CVPR'07. IEEE conference on. IEEE; 2007.

[16] Bregler Christoph, Malik Jitendra, Pullen Katherine. Twist based acquisition and tracking of animal and human kinematics. Int J Comput Vision 2004;56(3):179-94.

[17] Traver V Javier, et al. Human gesture recognition using three-dimensional integral imaging. JOSA A 2014;31(10):2312-20.

[18] Wu Chen, Aghajan Hamid, Kleihorst Richard. Real-time human posture reconstruction in wireless smart camera networks. Proceedings of the 7th international conference on Information processing in sensor networks. IEEE Computer Society; 2008.

[19] Gavrila Dariu M, Davis Larry S. 3-D model-based tracking of humans in action: a multi-view approach. Computer vision and pattern recognition, 1996. Proceedings CVPR'96, 1996 IEEE computer society conference on. IEEE; 1996.

[20] Ivekovic Spela, Trucco Emanuele. Human body pose estimation with pso. Evolutionary Computation, 2006. CEC 2006. IEEE congress on. IEEE; 2006.

[21] Sigal Leonid, et al. Tracking loose-limbed people. Computer vision and pattern recognition, 2004. CVPR 2004. Proceedings of the 2004 IEEE computer society conference on, vol. 1. IEEE; 2004.

[23] Miki Ivana, et al. Human body model acquisition and tracking using voxel data. Int J Comput Vision 2003;53(3):199-223.

[24] Sigal Leonid, Michael J Black. Predicting 3d people from 2d pictures. Articulated motion and deformable objects. Springer Berlin Heidelberg; 2006. p. 185-95.

[25] Lee Mun Wai, Cohen Isaac. Proposal maps driven mcmc for estimating human body pose in static images. Computer vision and pattern recognition, 2004. CVPR 2004. Proceedings of the 2004 IEEE computer society conference on, vol. 2. IEEE; 2004

[33] Hong Seung-Hyun, Jang Ju-Seog, Javidi Bahram. Three-dimensional volumetric object reconstruction using computational integral imaging. Opt Express 2004;12(3):483-91. 